\title{
TOPOLOGICAL PROPERTIES OF SUBSETS OF THE ZARISKI SPACE
}

\author{
DARIO SPIRITO \\ Dipartimento di Matematica e Fisica, Università degli Studi "Roma Tre" \\ Roma, Italy \\ ORCID: 0000-0002-7318-7860Ｅ-mail: spirito@mat.uniroma3.it
}

\begin{abstract}
We study the properties of some distinguished subspaces of the Zariski space $\operatorname{Zar}(K \mid D)$ of a field $F$ over a domain $D$, in particular the topological properties of subspaces defined through algebraic means. We are mainly interested in two classes of problems: understanding when spaces of the form $\operatorname{Zar}(K \mid D) \backslash\{V\}$ are compact (which is strongly linked to the problem of determining when $\operatorname{Zar}(K \mid D)$ is a Noetherian space), and studying spaces of rings defined through pseudo-convergent sequences on an (arbitrary, but fixed) rank one valuation domain.
\end{abstract}

1. Introduction and notation. Let $D$ be an integral domain and $K$ be a field containing $D$ (not necessarily the quotient field of $D$ ). In the Thirties, studying the problem of resolution of singularities, Zariski introduced the Zariski space of $K$ over $D$ (under the name generalized Riemann surface) as the set $\operatorname{Zar}(K \mid D)$ of all valuation domains of $K$ containing $D$ [23, 24]. He introduced on this set a topology (later called the Zariski topology) which is generated by the open sets

$$
\mathcal{B}\left(x_{1}, \ldots, x_{n}\right):=\left\{V \in \operatorname{Zar}(K \mid D) \mid x_{1}, \ldots, x_{n} \in V\right\},
$$

as $x_{1}, \ldots, x_{n}$ range in $K$, and showed that, under this topology, $\operatorname{Zar}(K \mid D)$ is a compact space [25, Chapter VI, Theorem 40].

Later, it was shown that $\operatorname{Zar}(K \mid D)$ is actually a spectral space (in the sense of Hochster [9]), that is, for every $K$ and $D$ there is a ring $R$ such that $\operatorname{Zar}(K \mid D) \simeq \operatorname{Spec}(R)$;

2010 Mathematics Subject Classification: Primary 13F30; Secondary 12J20, 13A15, 13A18, 54D30, 54E35.

Key words and phrases: Zariski space, Zariski topology, constructible topology, non-compact subspaces, Noetherian space, pseudo-convergent sequence, pseudo-limit.

The paper is in final form and no version of it will be published elsewhere. 
such an $R$ can also be constructed explicitly as a Bézout domain having quotient field $K(X)$ (called the Kronecker function ring of $K$ over $D$ ) [4, 5, 6]. As a spectral space, $\operatorname{Zar}(K \mid D)$ can also be endowed with the inverse topology (the topology generated by the complements of the open and compact subspaces of the original topology) and the constructible (or patch) topology (the topology generated by both the open and compact subspaces and their complements). These two topologies are both spectral (so, in particular, compact) and, more importantly, $\operatorname{Zar}(K \mid D)^{\text {cons }}$ (i.e., $\operatorname{Zar}(K \mid D)$ under the constructible topology) is an Hausdorff space, something that does not happen for the Zariski or the inverse topology unless $D$ is a field and $K$ is an algebraic extension, i.e., unless $\operatorname{Zar}(K \mid D)$ is just $\{K\}$. Of particular importance are the closed sets of $\operatorname{Zar}(K \mid D)^{\text {cons }}$ : they are called proconstructible subsets, and they are again spectral spaces (in the Zariski topology).

These three topologies are closely linked with the algebraic properties of the valuation domains, and in particular there is a connection between the topological properties of $X \subseteq \operatorname{Zar}(K \mid D)$ and the algebraic properties of the intersection of the elements of $X$ (called the holomorphy ring $A(X)$ of $X)$ [11, 12, 13, 14]: for example, if $X$ is a compact subset of one-dimensional valuation domains such that $\bigcap_{V \in X} \mathfrak{m}_{V} \neq(0)$, then $A(X)$ is a one-dimensional Bézout domain [14, Theorem 5.3]. In particular, for Prüfer domains, the set $\operatorname{Zar}(D)$ (that is, $\operatorname{Zar}(K \mid D)$ with $K$ being the quotient field of $D$ ) is homeomorphic to the spectrum of $D$ (under the Zariski topology). More generally, there is always a map $\gamma: \operatorname{Zar}(K \mid D) \longrightarrow \operatorname{Spec}(D), V \mapsto \mathfrak{m}_{V} \cap D$, called the center map, which is continuous ([25. Chapter VI, $\S 17$, Lemma 1] or [4, Lemma 2.1]), surjective (this follows, for example, from [1, Theorem 5.21] or [8, Theorem 19.6]) and closed [4, Theorem 2.5].

The space $\operatorname{Zar}(K \mid D)$ can also be considered as a subspace of the set $\operatorname{Over}(K \mid D)$ of the rings comprised between $D$ and $K$, as a subspace of the set of $D$-submodules of $K$ or, even more generally, as a subspace of the power set of $K$; all these sets become spectral spaces under the natural extension of the Zariski topology [3, 1.9.5(vi-vii)]. It is to be noted that a closer look at Zariski's proof of the compactness of $\operatorname{Zar}(K \mid D)$ actually shows that $\operatorname{Zar}(K \mid D)$ is a proconstructible subset of the power set $\mathcal{P}(K)$ [13, discussion after Proposition 2.1].

2. Compactness. In general, it is hard to find subsets of $\operatorname{Zar}(K \mid D)$ that are not compact. A general algebro-geometric criterion was given in [7, Lemma 5.8(2)] through the theory of semistar operations; to be useful, however, it has to be applied together with the theory of the $b$-operation/integral closure, which can be defined either as the semistar operation induced by the whole $\operatorname{Zar}(D)$ or through integral dependence of ideals [21]. The first consequence is the following.

Theorem 2.1 ([19, Proposition 7.1]). Let D be a Noetherian ring with quotient field $K$, and let $\Delta$ be the set of Noetherian valuation overrings of $D$. Then, $\Delta$ is compact if and only if $\operatorname{dim}(D) \leq 1$.

(Note that, when $\operatorname{dim}(D) \leq 1$, the set $\Delta$ is actually just $\operatorname{Zar}(D)$.) If $\Delta$ is as in the theorem, then we can write $\Delta=X(D) \cap \operatorname{Zar}(D)$, where $X(D)$ is the set of Noetherian overrings of $D$; in particular, $X(D)$ cannot be proconstructible in the Zariski topology of $\operatorname{Over}(D)$, since this would imply that $\Delta$, as the intersection of two proconstructible 
subspaces, is itself proconstructible. The same happens for other subsets of Noetherian rings.

Proposition 2.2 ([19, Proposition 7.3 and Corollary 7.7]). Let $D$ be a Noetherian domain. Then, the following are equivalent:

(i) $\operatorname{dim}(D)=1$;

(ii) $X(D)$ is compact;

(iii) the set $\{T \in \operatorname{Over}(D) \mid T$ is a Dedekind domain $\}$ is compact;

(iv) the set $\{T \in \operatorname{Over}(D) \mid T$ is Noetherian of dimension 1$\}$ is compact.

The same holds if "compact" is substituted with "proconstructible".

Another interesting case is the one in which we delete just one valuation domain.

Theorem 2.3 ([19, Theorem 3.6]). Let $D$ be an integral domain and $V$ be a minimal element of $\operatorname{Zar}(D)$. If $\operatorname{Zar}(D) \backslash\{V\}$ is compact, then $V$ is equal to the integral closure of $D\left[x_{1}, \ldots, x_{n}\right]_{M}$ for some $x_{1}, \ldots, x_{n} \in K$ and some $M \in \operatorname{Max}\left(D\left[x_{1}, \ldots, x_{n}\right]\right)$.

This condition is very strong; for example, it cannot happen in any of the following cases:

- $D$ is Noetherian and $\operatorname{dim}(V) \geq 2$;

- $\operatorname{dim}(V)>2 \operatorname{dim}(D)$ [19, Proposition 4.3];

- $D$ is local and $\bigcap\{P \mid P \in \mathcal{Y}\}=(0)$ for some family $\mathcal{Y}$ of nonzero incomparable prime ideals [19, Theorem 5.1].

A topological space $X$ is Noetherian if all its subsets are compact; equivalently, if the open sets of $X$ satisfy the ascending chain condition. For example, the prime spectrum of any Noetherian ring is a Noetherian space [1, Chapter 6, Exercises 5-8]. On the other hand, by either of the previous two cases, $\operatorname{Zar}(D)$ is not a Noetherian space as soon as $D$ is a Noetherian domain of dimension 2 or more. Indeed, the Noetherianity of $\operatorname{Zar}(K \mid D)$ is an extremely rare phenomenon.

Proposition 2.4. Let $D$ be an integral domain and let $K$ be a field containing $D$; suppose that $D$ is integrally closed in $K$.

(a) [20, Proposition 4.2] If $D=F$ is a field, then $\operatorname{Zar}(K \mid F)$ is a Noetherian space if and only if $\operatorname{trdeg}_{F} K \leq 1$ and, for every $T \in K$ transcendental over $F$, every valuation on $F[T]$ extends to finitely many valuations of $K$.

(b) [20, Theorem 5.11 and Corollary 5.12] If $D$ is local and not a field, then $\operatorname{Zar}(D)$ is Noetherian if and only if $D$ is a pseudo-valuation domain ${ }^{1} K$ is the quotient field of $D$ and $\operatorname{Zar}(L \mid F)$ is Noetherian, where $F$ is the residue field of $D$ and $L$ is the residue field of the associated valuation domain.

(c) [20, Theorem 5.11 and Corollary 5.12] If $D$ is not a field, then $\operatorname{Zar}(K \mid D)$ is Noetherian if and only if $K$ is the quotient field of $D, \operatorname{Spec}(D)$ is Noetherian and $\operatorname{Zar}\left(D_{M}\right)$ is Noetherian for every $M \in \operatorname{Max}(D)$.

\footnotetext{
${ }^{1} \mathrm{~A}$ pseudo-valuation domain (PVD) is a local domain $(D, \mathfrak{m})$ having a valuation overring $V$ whose maximal ideal is $\mathfrak{m}$; such $V$ is called the valuation domain associated to $D$.
} 
In particular, these domains have a fairly peculiar Zariski space: in the local case, the non-minimal valuations of $D$ are all comparable, and the valuative dimension of $D$ can be only $\operatorname{dim}(D)$ or $\operatorname{dim}(D)+1$ [20, Proposition 5.13].

3. Pseudo-convergent sequences. Let now $V$ be a one-dimensional valuation ring with valuation $v$, value group $\Gamma_{v} \subseteq \mathbb{R}$ and quotient field $K$. A pseudo-convergent sequence of $V$ is a sequence $E=\left\{s_{n}\right\}_{n \in \mathbb{N}} \subset K$ such that

$$
v\left(s_{n}-s_{n-1}\right)<v\left(s_{n+1}-s_{n}\right)
$$

for all $n \in \mathbb{N}, n \geq 1$. Pseudo-convergent sequences were introduced by Ostrowski to determine all the rank-one extensions of $V$ to $K(X)$ [15, 16], and subsequently used by Kaplansky to investigate maximal valued fields [10]. They can be generalized to pseudomonotone sequences [2, Definition 4.6].

The gauge of $E$ is the sequence of the $\delta_{n}:=v\left(s_{n+1}-s_{n}\right)$ [22, p. 327]; it is a strictly increasing sequence of real numbers, and its limit $\delta_{E} \in \mathbb{R} \cup\{\infty\}$ is called the breadth of $E$. In particular, $\delta_{E}$ is infinite if and only if $E$ is a Cauchy sequence (in the topology induced by the valuation). If $V$ is discrete, every pseudo-convergent sequence has infinite breadth. The ideal $\operatorname{Br}(E):=\left\{x \in V \mid v(x) \geq \delta_{E}\right\}$ is called the breadth ideal of $E$.

Pseudo-convergent sequences can be divided into two classes: $E$ is of algebraic type if $v\left(f\left(s_{n}\right)\right)$ is definitively increasing for some polynomial $f \in K[X]$, while it is of transcendental type otherwise [10, Definitions, p. 306]. If $v\left(\alpha-s_{n}\right)<v\left(\alpha-s_{n+1}\right)$ for all $n \in \mathbb{N}$ (or, equivalently, if $v\left(\alpha-s_{n}\right)=\delta_{n}$ ), then $\alpha$ is said to be a pseudo-limit of $E$; if $\alpha \in \bar{K}$ (the algebraic closure of $K$ ), then we can use the same definition once we fix an extension $u$ of $v$ to $\bar{K}$. In particular, $E$ is of algebraic type if and only if it has a pseudo-limit in $\bar{K}$ [10, Theorems 2 and 3]. Pseudo-limits are not unique, but if $\alpha$ is one of them, then the set $\mathcal{L}(E)$ of the pseudo-limits of $E$ is the coset $\alpha+\operatorname{Br}(E)$ [10, Lemma 3]. The name "algebraic" and "transcendental" derive from the fact that, if $E$ is a Cauchy sequence, the limit of $E$ in $\widehat{K}$ is algebraic (resp., transcendental) over $K$ if and only if $E$ is of algebraic (resp., transcendental) type.

To each pseudo-convergent sequence $E$ we associate the map $w_{E}: K(X) \longrightarrow \mathbb{R} \cup\{\infty\}$ such that [17, Propositions 4.3 and 4.4]

$$
w_{E}(\phi):=\lim _{n \rightarrow \infty} v\left(\phi\left(s_{n}\right)\right) .
$$

Then, $w_{E}$ is a valuation on $K(X)$ if $E$ is of transcendental type or if $E$ is of algebraic type and $\delta_{E}<\infty$; if $E$ is of algebraic type and $\delta_{E}=\infty$, then $w_{E}$ is only a pseudo-valuation ${ }^{2}$ If $w_{E}$ is a valuation, the corresponding valuation ring $W_{E}$ is a one-dimensional extension of $V$ to $K(X)$; if $K$ is algebraically closed, then every rank-one extension of $V$ to $K(X)$ is in this form [15, 16]. We denote the set of all rings in the form $W_{E}$ as $\mathcal{W}$ : then, the Zariski and the constructible topologies agree on $\mathcal{W}$, and under them $\mathcal{W}$ is a regular zero-dimensional space that is not compact [17, Propositions 6.3 and 6.4].

${ }^{2}$ A pseudo-valuation on $K$ is a map $v: K \longrightarrow \Gamma_{v} \cup\{\infty\}$ (where $\left(\Gamma_{v},+\right.$ ) is a totally ordered abelian group) such that $v(a+b) \geq \min \{v(a), v(b)\}$ and $v(a b)=v(a)+v(b)$ for all $a, b \in K$; that is, it is a valuation without the hypothesis that only 0 goes to $\infty$. It is not linked with the notion of pseudo-valuation domain used in Section 2 
To every pseudo-convergent sequence $E$ can be associated another valuation domain, defined as

$$
V_{E}:=\left\{\phi \in K(X) \mid \phi\left(s_{n}\right) \in V \text { for all large } n\right\} .
$$

The ring $V_{E}$ is always an extension of $V$ to $K(X)$, and it is contained in $W_{E}$ (if $W_{E}$ is defined). If $E$ is of transcendental type, then $V_{E}=W_{E}$ is an immediate extension of $E$ [17, Theorem 4.9(a)]. On the other hand, if $E$ is of algebraic type, then the value group of $V_{E}$ is always isomorphic to $\Gamma_{v} \oplus \mathbb{Z}$, and the rank of $V_{E}$ depends on the breadth [17, Theorem 4.9(b,c)]:

- if $k \delta \in \Gamma_{v}$ for some positive $k \in \mathbb{N}$, then $V_{E}$ has rank 2 and $W_{E}$ has rank 1;

- if $\delta<\infty$ and $k \delta \notin \Gamma_{v}$ for all positive $k \in \mathbb{N}$, then $V_{E}=W_{E}$ has rank 1;

- if $\delta=\infty$, then $V_{E}$ has rank 2 and its one-dimensional overring is $K[X]_{(q)}$, where $q$ is the minimal polynomial of the limit of $E$.

The valuation $v_{E}$ can also be described explicitly as a map into $\mathbb{R}^{2}$ (see [17. Theorem 4.10]).

We denote the set of all the $V_{E}$ as $\mathcal{V}$ : then, $\mathcal{V}$ is a regular space in both the Zariski and the constructible topologies [17, Theorem 6.15], but the two topologies agree on $\mathcal{V}$ if and only if the residue field of $V$ is finite [17, Proposition 6.11]. There is also a map

$$
\begin{gathered}
\mathcal{W} \longrightarrow \mathcal{V} \\
W_{E} \longmapsto V_{E}
\end{gathered}
$$

that, under the Zariski topology, is continuous and injective, but not a topological embedding [17, Proposition 6.13].

There are two natural ways to partition $\mathcal{V}$, either by fixing the breadth of the sequences or by fixing a pseudo-limit.

Let $\delta \in \mathbb{R} \cup\{\infty\}$, and define $\mathcal{V}(\bullet, \delta):=\left\{V_{E} \in \mathcal{V} \mid \delta_{E}=\delta\right\}$. Then, the Zariski and the constructible topologies agree on $\mathcal{V}(\bullet, \delta)$ [18, Theorem 3.5]; furthermore, this topology is also generated by the ultrametric distance

$$
d_{\delta}\left(V_{E}, V_{F}\right):=\lim _{n \rightarrow \infty} \max \left\{d\left(s_{n}, t_{n}\right)-e^{-\delta}, 0\right\},
$$

where $E:=\left\{s_{n}\right\}_{n \in \mathbb{N}}$ and $F:=\left\{t_{n}\right\}_{n \in \mathbb{N}}$. Under this metric, $\mathcal{V}(\bullet, \delta)$ is complete, and is the completion of the subspace [18, Proposition 3.4]

$$
\mathcal{V}_{K}(\bullet, \delta):=\left\{V_{E} \in \mathcal{V}(\bullet, \delta) \mid E \text { has a pseudo-limit in } K\right\}
$$

When $\delta=\infty$, the space $\mathcal{V}(\bullet, \infty)$ is canonically isomorphic to the completion $\widehat{K}$, and $d_{\infty}$ reduces to the distance induced by $\widehat{v}$; furthermore, $\mathcal{V}_{K}(\bullet, \infty)$ corresponds to $K$. Hence, $\mathcal{V}(\bullet, \delta)$ can be seen as a generalization of the completion of $V$, with the elements of $\mathcal{V}(\bullet, \delta)$ corresponding to the closed balls of $V$ of radius $e^{-\delta}$. Note that the various $d_{\delta}$ cannot be unified to a metric on the whole $\mathcal{V}$ (since otherwise they would define closed subspaces of $\mathcal{V}$, but the $\mathcal{V}(\bullet, \delta)$ are not closed) [18, Proposition 3.8].

Let $\beta \in \bar{K}$, fix an extension $u$ of $v$ to $\bar{K}$ and let

$$
\mathcal{V}^{u}(\beta, \bullet):=\left\{V_{E} \in \mathcal{V} \mid \beta \text { is a pseudo-limit of } E \text { w.r.t. } u\right\} \text {. }
$$


Then, each $\mathcal{V}^{u}(\beta, \bullet)$ is a closed subspace of $\mathcal{V}$ [18, Proposition 4.2], and the Zariski and the constructible topologies agree on $\mathcal{V}^{u}(\beta, \bullet)$ [18, Proposition 4.6]; furthermore, the elements of $\mathcal{V}^{u}(\beta, \bullet)$ are parametrized by the breadth, and so there is a bijection between $\mathcal{V}^{u}(\beta, \bullet)$ and $(-\infty, \delta(\beta, K)]$ (given by $\left.E \mapsto \delta_{E}\right)$, where $\delta(\beta, K):=\sup \{u(\beta-x) \mid x \in K\}$ represent (the valuation relative to) the distance between $\beta$ and $K$. The topology induced by $\mathcal{V}^{u}(\beta, \bullet)$ on $(-\infty, \delta(\beta, K)]$ is generated by the sets $(a, b]$, with $b \in \mathbb{Q} \Gamma_{v},[18$, Theorem 4.4]. This topology is metrizable if and only if $\Gamma_{v}$ is countable; in particular, we have the following.

Proposition 3.1 ([18, Corollary 4.8]). If $\Gamma_{v}$ is not countable, then $\operatorname{Zar}(K(X) \mid V)^{\text {cons }}$ is not metrizable.

To conclude, we list some open problems on the topological properties of $\mathcal{V}, \mathcal{W}$ and their subsets.

- Is $\mathcal{V}$ zero-dimensional?

- Is $\mathcal{V}$ a normal space?

- Are $\mathcal{V}\left(\delta_{1}, \bullet\right)$ and $\mathcal{V}\left(\delta_{2}, \bullet\right)$ homeomorphic for $\delta_{1} \neq \delta_{2}$ ? (This is true if $\delta_{1}-\delta_{2} \in \Gamma_{v}$, 18, Proposition 3.9].)

- If $\Gamma_{v}$ is countable, are $\mathcal{V}$ and $\mathcal{W}$ metrizable?

- If $\Gamma_{v}$ is countable, is $\operatorname{Zar}(K(X) \mid V)^{\text {cons }}$ metrizable?

- More generally, when is $\operatorname{Zar}(K \mid D)^{\text {cons }}$ metrizable?

- If any of them is metrizable, can we find an ultrametric distance?

- What happens to $\mathcal{V}$ when the rank of $V$ is not 1 ?

\section{References}

[1] M. F. Atiyah, I. G. Macdonald, Introduction to Commutative Algebra, Addison-Wesley, Reading, Mass., 1969.

[2] J.-L. Chabert, On the polynomial closure in a valued field, J. Number Theory 130 (2010), $458-468$.

[3] J. Dieudonné, A. Grothendieck, Éléments de géométrie algébrique. IV. Étude locale des schémas et des morphismes de schémas. I, Inst. Hautes Études Sci. Publ. Math. 20 (1964), $5-259$.

[4] D. E. Dobbs, R. Fedder, M. Fontana, Abstract Riemann surfaces of integral domains and spectral spaces, Ann. Mat. Pura Appl. (4) 148 (1987), 101-115.

[5] D. E. Dobbs, M. Fontana, Kronecker function rings and abstract Riemann surfaces, J. Algebra 99 (1986), 263-274.

[6] C. A. Finocchiaro, M. Fontana, K. A. Loper, The constructible topology on spaces of valuation domains, Trans. Amer. Math. Soc. 365 (2013), 6199-6216.

[7] C. A. Finocchiaro, M. Fontana, D. Spirito, Spectral spaces of semistar operations, J. Pure Appl. Algebra 220 (2016), 2897-2913.

[8] R. Gilmer, Multiplicative Ideal Theory, Pure and Appl. Math. 12, Marcel Dekker, New York, 1972.

[9] M. Hochster, Prime ideal structure in commutative rings, Trans. Amer. Math. Soc. 142 (1969), 43-60. 
[10] I. Kaplansky, Maximal fields with valuations I, Duke Math. J. 9 (1942), 303-321.

[11] B. Olberding, Noetherian spaces of integrally closed rings with an application to intersections of valuation rings, Comm. Algebra 38 (2010), 3318-3332.

[12] B. Olberding, Affine schemes and topological closures in the Zariski-Riemann space of valuation rings, J. Pure Appl. Algebra 219 (2015), 1720-1741.

[13] B. Olberding, Topological aspects of irredundant intersections of ideals and valuation rings, in: Multiplicative Ideal Theory and Factorization Theory, Springer Proc. Math. Stat. 170, Springer, Cham, 2016, 277-307.

[14] B. Olberding, A principal ideal theorem for compact sets of rank one valuation rings, J. Algebra 489 (2017), 399-426.

[15] A. Ostrowski, Untersuchungen zur arithmetischen Theorie der Körper. Teil I, Math. Z. 39 (1935), 269-320.

[16] A. Ostrowski, Untersuchungen zur arithmetischen Theorie der Körper. Teil II und III, Math. Z. 39 (1935), 321-404.

[17] G. Peruginelli, D. Spirito, The Zariski-Riemann space of valuation domains associated to pseudo-convergent sequences, Trans. Amer. Math. Soc. 373 (2020), 7959-7990.

[18] G. Peruginelli, D. Spirito, Metrizability of spaces of valuation domains associated to pseudoconvergent sequences, submitted.

[19] D. Spirito, Non-compact subsets of the Zariski space of an integral domain, Illinois J. Math. 60 (2016), 791-809.

[20] D. Spirito, When the Zariski space is a Noetherian space, Illinois J. Math. 63 (2019), 299316.

[21] I. Swanson, C. Huneke, Integral Closure of Ideals, Rings, and Modules, London Math. Soc. Lecture Note Ser. 336, Cambridge Univ. Press, Cambridge, 2006.

[22] S. Warner, Topological Fields, North-Holland Math. Stud. 157, Notas de Matemática 126, North-Holland, Amsterdam, 1989.

[23] O. Zariski, The reduction of the singularities of an algebraic surface, Ann. of Math. (2) 40 (1939), 639-689.

[24] O. Zariski, The compactness of the Riemann manifold of an abstract field of algebraic functions, Bull. Amer. Math. Soc. 50 (1944), 683-691.

[25] O. Zariski, P. Samuel, Commutative Algebra, Vol. II, Grad. Texts in Math. 29, Springer, New York, 1975. 
